\title{
INCREASING OBESITY AND ITS RISK FACTORS
}

\author{
Maria Altaf ${ }^{2} \&$ Kisa Fatima ${ }^{2}$ \\ 1. Fatima Jinnah Dental College \\ 2. DOW University of Health Sciences
}

Corresponding author: maria3188@gmail.com

\begin{abstract}
Obesity has been a target tool for research for its prevention and treatment because of its association with various metabolic dysfunctions. Increased adipose tissue distribution leads to progressive increase in hypertension and cardiovascular disease prevalence. It was a prospective study conducted at Fatima Jinnah Dental College and Dow Institute of Medical Technology (DUHS) Karachi, from February 2012 to January 2013Seventy five subjects (54 girls and 21 boys) were randomly selected of ages 18-25 years.BMI, waist hip ratio and neck circumference was observed to determine obesity. Questionnaires were administered to ascertain biographical data, lifestyles behaviors including dietary habits and diet plan (healthy food/junk food), binge eating, family history for obesity, habits of cigarette smoking and alcohol consumption, patterns of daily physical activity. According to the anthropometric measurements out of seventy five individuals $20 \%$ were underweight, $34.7 \%$ were normal, $21 \%$ overweight, $17 \%$ obese and $6.7 \%$ were morbidly obese. $32 \%$ of people are mostly dependant on junk food, $45.2 \%$ people are occasionally dependant on junk food and only $22.7 \%$ people rely on homemade food, $36 \%$ individuals were physically active and $64 \%$ people were leading sedentary life style lack of time due to their academic schedule or lack of interest. In this context, two main factors appear to participate in body-weight maintenance: dietary habits and physical activity. Effective prevention and management of obesity requires an integrated approach, with intervention across different segments of the population through adequate information and public awareness programs.
\end{abstract}

\section{KEYWORDS}

Hypertension, anthropometric, hyperinsulinemia, diabetes, hypertriglyceridemia, gout, uric calculus, dysfunctions

\section{PURPOSE OF STUDY}

To determine the factors which causes increasing obesity in young adults and its effects on health.

\section{INTRODUCTION}

Obesity has become a leading health problem in Asian countries and it has increased consistently in past few years. It has been a target tool for research for its prevention and treatment because of its association with various diseased conditions (Kuk, 2007). Recently it is observed that adults are being more prone to the incidence of hypertension and other cardiovascular risk factors (Burke, 2006). Increased adipose tissue distribution and its relation among nutritional factors, homodynamic and hormonal imbalance leads to progressive increase in hypertension and cardiovascular disease prevalence (CoatmellecTaqlioni, 2003). Visceral and posterior subcutaneous fat deposition in adipose tissues are highly inter related with metabolic dysfunctions (Chan, 2003). Excess of visceral fat deposition decreases plasma level of adiponectin which is secreted as adipocytokines by adipose tissue having adverse effects on inflammatory, oncogenic, diabetic and artherogenic factors (Li, 2006). Obesity is highly associated with various metabolic dysfunctions, considering neck circumference upper body obesity may lead to glucose intolerance, hyperinsulinemia, diabetes, hypertriglyceridemia, gout, and uric calculus disease (Vague, 1956; Kissebah, 1982). Sjöström et al evaluated neck circumference as an index for measurement of upper body obesity and its relation between Cardiovascular risk factors and adipose tissue deposition (Sjöström, 1995). Low body obesity has been linked to increased risk of coronary heart disease, type II diabetes mellitus, cancer and all cause mortality (Folsom, 2000; Vazquez, 2007).Calories- the form of energy which is to be burnt in daily physical activity, main source of this energy is food we eat.
Body weight is increased when this energy remains stored in our body unutilized and is deposited in body as fat tissue (Hesketh, 2005). Anthropometric measurements especially BMI serves as an important and inexpensive tool for measurement of body fat with an exception in athletes for there large bone and muscle mass (Deurenberg, 1999). Energy expenditure is done by physical activity of skeletal muscles. Person being more physically active expands more stored energy from body. Physical activity vary from person to person as it completely depends upon personal intrest, however it is to be done to maintain proper healthy life (Caspersen, 1985). Physical activity and active lifestyle have beneficial effects on reducing cardiovascular risk factors in healthy individuals (Berlin, 1990). Physical activity raises high-density lipoprotein cholesterol, lowers low-density lipoprotein cholesterol and triglycerides, lowers blood pressure, improves fasting and postprandial glucose insulin homeostasis, induces and maintains weight loss, improves psychological well-being, and likely lowers inflammation, improves endothelial function, and facilitates smoking cessation (Thompson, 2003; Netz, 2005 \& Bassuk, 2005). Exercise also have remarkable effects on brains molecular systems(as was widely assumed in the early 1990s) (Cotman, 2002).

\section{METHODOLOGY}

It was a prospective study conducted at Fatima Jinnah Dental College and Dow Institute of Medical Technology (DUHS) Karachi, from February 2012 to January 2013 Seventy five subjects (54 girls and 21 boys) were randomly selected of ages 18-25 years belonging to different socio-economic group. The data was analyzed by statistical software SPSS version 17 and well designed questionnaires were administered to ascertain biographical data, lifestyles behaviors including dietary habits and diet plan (healthy food/junk food), binge eating, family history for obesity, habits of cigarette smoking and alcohol consumption, patterns of daily physical activity. 
A digital scale was used to measure Body Weight (BW) with accuracy; Subjects were weighed without shoes, in their normal clothing. Standing Body Height (BH) was measured without shoes to the nearest $0.5 \mathrm{~cm}$ with the use of a commercial stadiometer with the shoulders in the relaxed position and arms hanging freely. BMI was calculated as $\mathrm{BW}$ in kilograms $(\mathrm{Kg})$ divided by square of the $\mathrm{BH}$ in meters (m2). Based on WHO recommendations for Asian population a person was said to be underweight with $\mathrm{BMI}<18.5$, normal weight between 18.5-22.9, overweight between 23-24.9, obese type-1: between 25-29.9, and type II obese >30.15. Waist Circumference (WC) was measured in the middle between 12th rib and iliac crest and the Hip circumference ( $\mathrm{HC})$ at the fullest point around the buttocks. WC $(\mathrm{cm})$ was divided by $\mathrm{HC}(\mathrm{cm})$ in order to calculate the waist to hip ratio pulse rate was observed for one minute using pulse ox meter , subjects were seated in a chair with their back supported and their arms rested at heart level. Measurement was performed with the subject not having ingested coffee or smoked for 30 minutes and after at least five minutes of rest. Then the subject was asked to perform step up exercise on bench of 12 inches height for three minutes on metronome which was kept on 46 for females and 49 for males, each minute after exercise pulse rate was observed with help of pulse ox meter to determine the recovery period

\section{RESULT}

The mean age, height, $\mathrm{NC}$, and hip circumferences were similar for men and women. In both sexes, BMI correlated positively with waist circumference, hip circumference, and NC and waist: hip ratio. In bivariate analysis, a strong correlation was found between $\mathrm{NC}$ and age, weight, waist circumference, waist: hip ratio and BMI for men and women. According to the anthropometric measurements out of seventy five individuals $20 \%$ were underweight, $34.7 \%$ were normal, $21 \%$ overweight, $17 \%$ obese and $6.7 \%$ were morbidly obese. A questionnaire was circulated among these individuals which showed that $36 \%$ individuals actively takes part physical activity for more than 30 minutes a day and 64\% people can't take part because of lack of time due to their academic schedule or lack of interest. Dietary habits were analyzed which represents $32 \%$ of people are mostly dependant on junk food, $45.2 \%$ people are occasionally dependant on junk food and only $22.7 \%$ people rely on homemade food. No relation of obesity was found between smoking and alcohol consumption.

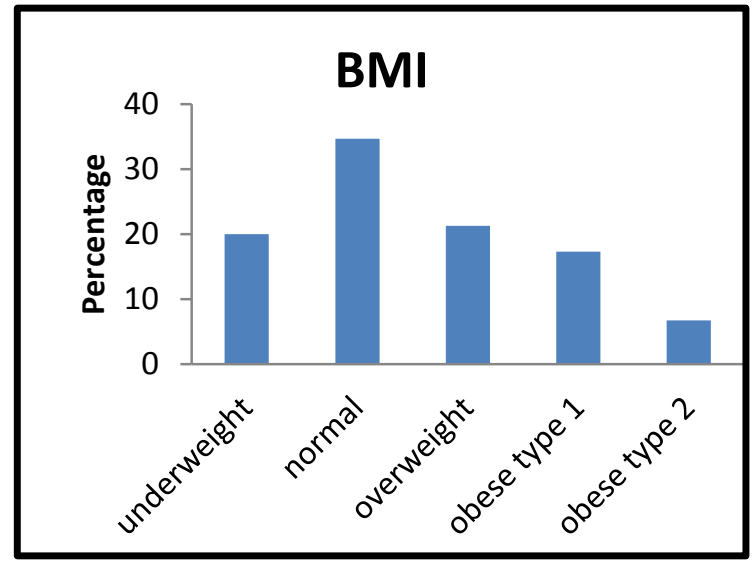

Figure\# 1 showing BMI

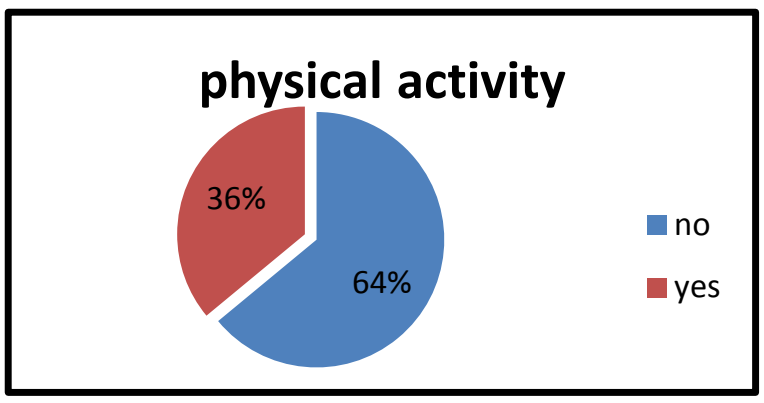

Figure\# 2 showing $\%$ of physical activity

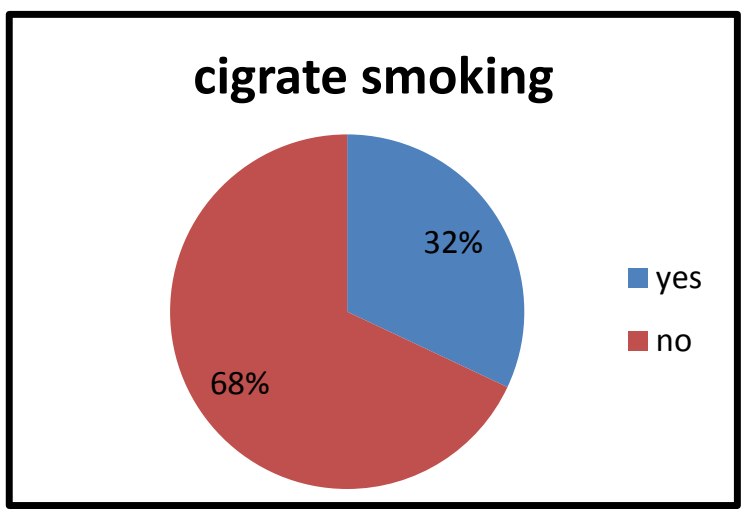

Figure\# 3 showing individual addiction to cigarette smoking

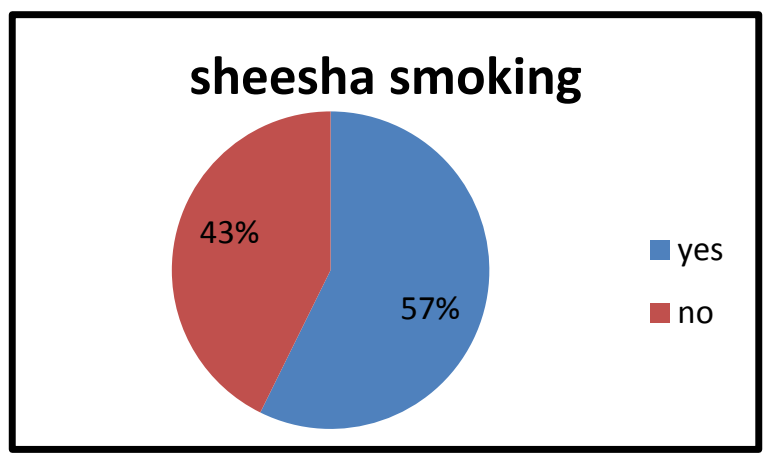

Figure\# 4 showing sheeshe smoking

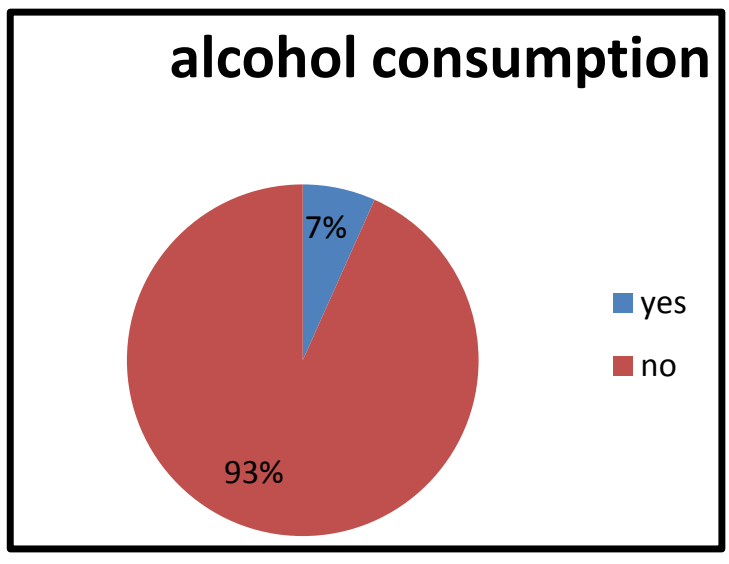

Figure\# 5 showing alcohol consumption 


\section{DISCUSSION}

Waist circumference and waist hip ratio are two simple and convenient ways to measure stored body fat. Many studies revealed waist circumference as a best measure of excess visceral fat, which can independently predict health risk when BMI is not markedly elevated. Whereas, other studies suggest waist hip ratio as a sensitive predictor of disease, as it assesses the risk of hypertension in a population when majority is considered thin as traditionally measured by BMI. (Fuchs, 2005)

Weight gain also depends on the distribution of dietary energy substrates, which have different impacts on metabolism and food intake, as well as on the sympathetic nervous system and, thereby, on energy balance and body weight (Prentice, 1998). The interactions between heredity and sedentariness have been assessed using twin pairs, and it was concluded that the genetic background may modify the effect of physical activity on weight change in males and females (Heitmann, 1997; Samaras 1999), and that lifestyle may have an obesity-promoting effect, depending on a genetic predisposition (Hill, 1998a; Hill, 1998b).

Subjects involved in this study were mainly students having a sedentary life style. When they were questioned for the reason answer was, they get less time for physical activity due their academic schedule. Regardless of the measure, these studies indicated that an increase in the amount of time dedicated towards physical healthbased activities (such as physical education) is not accompanied by a decline in academic performance. The implications of these findings are important for promoting better physical health.

People eat even when they are not hungry: in front of the television, in social occasions, and as a way of dealing with loneliness, boredom, anger, or other emotional stress. This type of eating is usually composed of snacks, which are high in calories contributes to obesity. Subjects having family history of obesity are mainly concern about their physical fitness as compare to those having non obese family history

It is well established that more active individuals weigh less than those who are sedentary. This relationship is supported by extensive epidemiologic research and is confirmed by a great deal of experimental research on humans and animals. A substantial proportion of subjects were overweight and obese, reflecting the general obesity trend in our society and who had higher levels of BP.

\section{CONCLUSION}

The accuracy of body-weight maintenance is achieved by a number of integrated homeostatic systems which adjust or match the energy balance constituents (energy intake versus energy expenditure). In this context, two main factors appear to participate in body-weight maintenance: dietary habits and physical activity. In this context, current epidemiological trends in weight for height measurements indicate that a major cause of the obesity problem lies in dietary and physical activity patterns, while genetic and metabolic studies reveal that there are individuals who are more susceptible to weight gain than others. Effective prevention and management of obesity requires an integrated approach, with intervention across different segments of the population through adequate information and public awareness programs. The examination of factors such as genetics and lifestyle implicated in weight gain and obesity is crucial for predictions about the future impact of the epidemic of obesity, and provides a unique opportunity for the implementation of preventive action.

\section{REFFRENCES}

- Kuk , J. L. (2007) Association between abdominal adiposity, exercise, morbidity and mortality. Appl Physiol Nutr Metab 32:1210-1.

- $\quad$ Burke, V.(2006) Obesity in childhood and cardiovascular risk. Clin Exp Pharmacol Physiol 33:831-7.

- Coatmellec-Taqlioni, G., \& Ribiere, C. (2003)Factors that influence the risk of hypertension in obese individuals. Curr Opin Nephrol Hypertens 12:305-8.

- $\quad$ Chan, D. C., Watts, G. F., Barrett, P. H. R., \& Burke, V. (2003). Waist circumference, waist-to-hip ratio and body mass index as predictors of adipose tissue compartments in men. Qjm, 96(6), 441-447.

- $\quad$ Li, C., Ford, E. S., Mokdad, A. H., \& Cook, S. (2006). Recent trends in waist circumference and waist-height ratio among US children and adolescents.Pediatrics, 118(5), e1390-e1398.

- Hesketh, K., Waters, E., Green, J., Salmon, L., \& Williams, J. (2005). Healthy eating, activity and obesity prevention: a qualitative study of parent and child perceptions in Australia. Health promotion international, 20(1), 19-26.

- Deurenberg, P., \& Yap, M. (1999). The assessment of obesity: methods for measuring body fat and global prevalence of obesity. Best Practice \& Research Clinical Endocrinology \& Metabolism, 13(1), 1-11.

- Vague, J. (1956). The degree of masculine differentiation of obesities a factor determining predisposition to diabetes, atherosclerosis, gout, and uric calculous disease. The American journal of clinical nutrition, 4(1), 20-34.

- $\quad$ Kissebah, A. H., Vydelingum, N., MURRAY, R., EVANS, D. J., KALKHOFF, R. K., \& ADAMS, P. W. (1982). Relation of body fat distribution to metabolic complications of obesity. Journal of Clinical Endocrinology \& Metabolism,54(2), 254-260.

- Sjöström, C. D., Håkangård, A. C., Lissner, L., \& Sjöström, L. (1995). Body Compartment and Subcutaneous Adipose Tissue Distribution-Risk Factor Patterns in Obese Subjects. Obesity research, 3(1), 9-22.

- Caspersen, C. J., Powell, K. E., \& Christenson, G. M. (1985). Physical activity, exercise, and physical fitness: definitions and distinctions for health-related research. Public health reports, $100(2), 126$.

- $\quad$ Berlin, J.A, \& Colditz, G.A.(1990) Ameta-analysis ofphysical activity in the prevention of coronary heart disease. Am J Epidemiol 312:612-27.

- Thompson, P. D., Buchner, D., Piña, I. L., Balady, G. J., Williams, M. A., Marcus, B. H., ... \& Wenger, N. K. (2003). Exercise and Physical Activity in the Prevention and Treatment of Atherosclerotic Cardiovascular Disease A Statement From the Council on Clinical Cardiology (Subcommittee on Exercise, Rehabilitation, and Prevention) and the Council on Nutrition, Physical Activity, and Metabolism (Subcommittee on Physical Activity). Circulation, 107(24), 3109-3116.

- $\quad$ Netz, Y., Wu, M. J., Becker, B. J., \& Tenenbaum, G. (2005). Physical activity and psychological well-being in advanced age: 
a meta-analysis of intervention studies. Psychology and aging, 20(2), 272.

- Bassuk, S. S., \& Manson, J. E. (2005). Epidemiological evidence for the role of physical activity in reducing risk of type 2 diabetes and cardiovascular disease.Journal of Applied Physiology, 99(3), 1193-1204.

- Cotman, C. W., \& Berchtold, N. C. (2002). Exercise: a behavioral intervention to enhance brain health and plasticity. Trends in neurosciences, 25(6), 295-301.

- Fuchs, F. D., Gus, M., Moreira, L. B., Moraes, R. S., Wiehe, M., Pereira, G. M., \& Fuchs, S. C. (2005). Anthropometric indices and the incidence of hypertension: a comparative analysis. Obesity Research, 13(9), 1515-1517.

- Bray, G. A. (1987). Obesity-A Disease of Nutrient or Energy Balance?. Nutrition reviews, 45(4), 33-43.

- Folsom, A. R., Kushi, L. H., Anderson, K. E., Mink, P. J., Olson, J. E., Hong, C. P., ... \& Prineas, R. J. (2000). Associations of general and abdominal obesity with multiple health outcomes in older women: the Iowa Women's Health Study. Archives of Internal Medicine, 160(14), 2117.

- Vazquez, G., Duval, S., Jacobs, D. R., \& Silventoinen, K. (2007). Comparison of body mass index, waist circumference, and waist/hip ratio in predicting incident diabetes: a metaanalysis. Epidemiologic Reviews, 29(1), 115-128.

- Canoy, D. (2008)Distribution of body fat and risk of coronary heart disease in men and women. Curr Opin Cardiol. (23)591598.

- $\quad$ Prentice, A.M. (1998) Manipulation of dietary fat and energy density and subsequent effects on substrate flux and food intake. American Journal of Clinical Nutrition (67) 535S-541S.

- Heitmann, B. L., Kaprio, J., Harris, J. R., Rissanen, A., Korkeila, M., \& Koskenvuo, M. (1997). Are genetic determinants of weight gain modified by leisure-time physical activity? A prospective study of Finnish twins. The American journal of clinical nutrition, 66(3), 672-678.

- Samaras, K., Kelly, P. J., Chiano, M. N., Spector, T. D., \& Campbell, L. V. (1999). Genetic and environmental influences on total-body and central abdominal fat: the effect of physical activity in female twins. Annals of Internal Medicine, 130(11), 873-882.

- Hill, J.O. (1998) Genetic and environmental contributions to obesity. American Journal of Clinical Nutrition (68) 991-992.

- Hill, J.O. \& Peters. J.C. (1998) Environmental contributions to the obesity epidemic. Science (280) 1371-1374. 